\title{
The gait and balance of patients with diabetes can be improved: a randomised controlled trial
}

\author{
L. Allet • S. Armand • R. A. de Bie • A. Golay • \\ D. Monnin • K. Aminian • J. B. Staal • E. D. de Bruin
}

Received: 28 June 2009 / Accepted: 6 October 2009 / Published online: 17 November 2009

(C) The Author(s) 2009. This article is published with open access at Springerlink.com

\begin{abstract}
Aims/hypothesis Gait characteristics and balance are altered in diabetic patients. Little is known about possible treatment strategies. This study evaluates the effect of a specific training programme on gait and balance of diabetic patients.
\end{abstract}

Electronic supplementary material The online version of this article (doi:10.1007/s00125-009-1592-4) contains supplementary material, which is available to authorised users.

L. Allet $\cdot$ R. A. de Bie $\cdot$ J. B. Staal

Department of Epidemiology,

Maastricht University and Caphri Research School,

Maastricht, the Netherlands

L. Allet $(\bowtie) \cdot$ D. Monnin

Care Services Directorate,

Unit of Physiotherapy Research and Quality Assurance,

Geneva University Hospital and University of Geneva,

Rue Gabrielle-Perret-Gentil 4,

1211 Geneva 14, Switzerland

e-mail: lara.allet@hcuge.ch

S. Armand

Willy Taillard Laboratory of Kinesiology,

Geneva University Hospital and University of Geneva,

Geneva, Switzerland

\section{A. Golay}

Service of Therapeutic Education for Chronic Diseases,

WHO Collaborating Center,

Geneva University Hospital and University of Geneva,

Geneva, Switzerland

\section{K. Aminian}

Laboratory of Movement Analysis and Measurement, EPFL,

Lausanne, Switzerland

\section{E. D. de Bruin}

Institute of Human Movement Sciences and Sport, ETH,

Zürich, Switzerland
Methods This was a randomised controlled trial $(n=71)$ with an intervention $(n=35)$ and control group $(n=36)$. The intervention consisted of physiotherapeutic group training including gait and balance exercises with functionorientated strengthening (twice weekly over 12 weeks). Controls received no treatment. Individuals were allocated to the groups in a central office. Gait, balance, fear of falls, muscle strength and joint mobility were measured at baseline, after intervention and at 6-month follow-up.

Results The trial is closed to recruitment and follow-up. After training, the intervention group increased habitual walking speed by $0.149 \mathrm{~m} / \mathrm{s}(p<0.001)$ compared with the control group. Patients in the intervention group also significantly improved their balance (time to walk over a beam, balance index recorded on Biodex balance system), their performance-oriented mobility, their degree of concern about falling, their hip and ankle plantar flexor strength, and their hip flexion mobility compared with the control group. After 6 months, all these variables remained significant except for the Biodex sway index and ankle plantar flexor strength. Two patients developed pain in their Achilles tendon: the progression for two related exercises was slowed down.

Conclusions/interpretation Specific training can improve gait speed, balance, muscle strength and joint mobility in diabetic patients. Further studies are needed to explore the influence of these improvements on the number of reported falls, patients' physical activity levels and quality of life.

Trial registration: ClinicalTrials.gov NCT00637546

Funding: This work was supported by the Swiss National Foundation (SNF): PBSKP-123446/1/

Keywords Balance - Clinical diabetes - Diabetic foot . Exercise - Fear - Gait disorders · Joint mobility - Neuropathy Muscle strength $\cdot$ Walking 


\section{Abbreviations}

CVGCT Coefficient of variation of gait cycle time

FES-I Falls Efficacy Scale International

POMA Performance-oriented mobility assessment

\section{Introduction}

Type 2 diabetes mellitus and its common complication, peripheral neuropathy, affect a large population $[1,2]$. Peripheral neuropathy leads to sensory and motor deficits, which often result in mobility-related dysfunction, alterations in gait characteristics [3, 4] and balance impairments $[5,6]$. Diabetic patients with peripheral neuropathy have lower gait velocity, decreased cadence, shorter stride length, increased stance time and higher step to step variability compared with healthy controls [3]. These gait alterations increase on irregular surfaces [3]. Moreover, these patients have less ankle moment and ankle power [7,8], as well as a different onset and cessation time of muscle activity compared with healthy controls [7]. Patients present more co-contractions of agonist and antagonist muscles at the ankle and knee joints during stance phase. Kwon et al. [7] speculate that the co-contraction mechanism may enable these individuals to adopt a safer, more stable gait pattern to compensate for diminished sensory information. The same authors have reported reduced ankle strength and mobility, which they considered to be the primary factors contributing to gait alterations. Allet et al. [3] have also found lower limb strength, fear of falling and sensory problems to be related to spatiotemporal gait alterations. Additionally, individuals with peripheral neuropathy show postural instability with a larger centre of pressure displacement [9], higher sway area [10] and greater instability [11] when standing still with eyes closed [9]. Postural instability was further found to be significantly associated with sensory neuropathy [12]. In addition to these gait and balance impairments, diabetic patients are known to suffer from increased risk of injurious falls [13]. Fall-related injuries are often assumed to trigger a vicious circle because of their potentially detrimental influence on the physical activity levels of affected patients. Public Health guidelines for diabetes management recommend that patients perform at least $30 \mathrm{~min}$ of physical activity a day six times a week, requiring adequate gait security and balance. However, little is known about treatment strategies that could improve patients' gait and balance, thereby also reducing the risk of falls [4]. Although there is evidence that an exercise regimen improves clinical measures of balance in patients with peripheral neuropathy [14], clinical trials investigating the gait of diabetic individuals generally focus on increased foot pressure, another major problem in this population and related to the high risk of ulcers in such patients [15]. Only few studies have evaluated treatments that aim to improve gait and balance and decrease fall risk [16, 17]. Petrofsky et al. [16] tested an insulin sensitiser, rosiglitazone, which promises to reverse some of the circulatory impairments seen in diabetes, thereby improving patients' gait. They reported encouraging results after administering rosiglitazone (decreased step width, reduced reaction time and less acceleration at the joints). However, rosiglitazone was recently associated with increased risk of myocardial infarction and death from cardiovascular incidents [18]. Richardson et al. [17], evaluating patients with various form of peripheral neuropathy (30 of 42 of whom had diabetic peripheral neuropathy), found that the use of a cane, ankle orthoses or touching a wall improved step-width range, step-time variability and speed while walking under challenging conditions. To the best of our knowledge, only Tsang et al. [19] and Orr et al. [20] have investigated the effect of a specific physical training programme not only on the activity level and quality of life of diabetic patients, but also on their habitual and maximal walking speed. However, both studies seem to have evaluated the same group of participants. In these studies, the effect of a 'Tai Chi for Diabetes' programme (twice a week for 16 weeks) on gait, balance, musculoskeletal and cardiovascular fitness, self-reported activity and quality of life was compared with that of sham exercises. Gait speed and balance improved, but no significant differences between groups were reported. Nevertheless, several studies [21-23] have shown positive training effects on gait speed, postural stability and mobility of elderly individuals. Additionally, a meta-analysis [24] evaluating fall prevention studies for the elderly showed a $4 \%$ decrease in the fall-rate of individuals who were in a treatment group. Since elderly people often show symptoms similar to those in diabetic patients (i.e. deconditioning, muscle weakness, decreased joint mobility and decreased foot sensibility), we assumed that programmes developed for the elderly might also improve gait and balance of diabetic patients. Thus, this study aimed to evaluate the effect of a specific training programme, which was based on previously elaborated core components of successful fall prevention programmes for the elderly [25], on the gait and balance of diabetic patients.

Gait speed and coefficient of variation of gait cycle time (CVGCT) were selected as primary outcomes because they have been shown to be related to increased risk of falls [22, 26] (slow gait speed and high CVGCT indicate increased fall risk). Balance control, muscle strength and joint mobility are also important fall risk factors that may be influenced by exercise [27]. For these reasons, they were chosen as secondary outcomes. Fear of falls is a cognitive behavioural component, which was recently shown to be related to the gait velocity of diabetic patients [28]. It therefore completed our outcome list. 
We hypothesised that diabetic patients participating in a training programme would significantly improve their walking speed and gait variability (CVGCT). We further assumed that such patients would significantly: (1) improve their balance; (2) increase their lower limb strength (in particular hip extensors, knee extensors and ankle flexors); (3) increase their ankle joint mobility; and (4) decrease their fear of falling.

\section{Methods}

Study design This randomised controlled prospective trial with two arms (intervention group and control group) was conducted at the University Hospital of Geneva, Switzerland. The study was approved by the local Ethics Committee.

A sample of 71 diabetic patients was recruited from patients consulting either the Service of Therapeutic Education for Chronic Diseases or the Service of Endocrinology at the University Hospital in Geneva. Of these, 35 patients were allocated to the intervention group and 36 to the control group. Patients were included if they were medically diagnosed with type 2 diabetes (fasting blood sugar $\geq 7 \mathrm{mmol} / \mathrm{l}$ ). Only patients without medical contraindications for engaging in physical activity and with a clinically diagnosed neuropathy were enrolled. Clinically diagnosed neuropathy was evaluated on the basis of a vibration perception threshold of $\leq 4$ with a Rydel-Seiffer tuning fork [29]. Patients were requested to indicate when they could no longer feel the vibration. At this point the investigator rated the vibration on a 9 -point scale $(0=$ severe neuropathy; $8=$ no neuropathy). Exclusion criteria were: patients with concomitant foot ulcers, orthopaedic or surgical problems affecting gait variables, non-diabetic neuropathy (due to Charcot-Marie-Tooth disease or alcohol), other neurological pathologies (other than peripheral neuropathy) that could influence gait variables or inability to walk a minimum of $500 \mathrm{~m}$ without a walking aid.

Training programmes started as soon as enough patients (20 to 28 per group) had been recruited, i.e. in September 2007, January 2008 and April 2008. For each of these starting points a new randomisation list was electronically generated and was used by a person not involved in the recruitment, evaluation or treatment processes. Each list ensured equal distribution over groups.

All eligible patients identified by the medical staff were asked to participate. They were informed that if willing to participate, they would be randomly assigned to an intervention group or control group. Patients who agreed to join the study were contacted by the study coordinator for an individual appointment, at which the informed consent document was signed and baseline evaluation performed. After this initial appointment, during which patients underwent a clinical examination, a gait analysis and both static and dynamic balance tests, and also filled in a fear of falls questionnaire, patients were randomly allocated to the intervention group or to the control group. The allocation was accomplished by a secretary in a central office. All outcome measures were assessed at baseline, after 12 weeks and after 6 months by the same experienced physiotherapist each time.

Patients allocated to the intervention group received a timetable containing all planned sessions over 12 weeks. The programme started within ten working days after the examination. Patients were kept unaware of the study hypothesis. However, the nature of the study made it impossible to blind patients and therapists. The assessor was kept as much as possible unaware of the group assignment. However, as she worked in this hospital during the experiment, she saw some patients arriving for training sessions.

After the 12 week programme patients were encouraged to continue with the learned exercises during the next 6 months. No other advice or restrictions were provided.

Test description and measures Prior to the clinical examination, the physiotherapist checked the vibration perception threshold with the tuning fork. Maximum isometric strength of the hip, knee, ankle flexors and extensors was then measured with a hand-held dynamometer (MicroFET; Hoggan Health Industries, Inc., West Jordan, UT, USA) [30]. Joint mobility of the hip and both knee and ankle flexion and extension were measured with a manual goniometer. Patients' worry of falling when performing different activities was assessed with the Falls Efficacy Scale International (FES-I) [31]. Afterwards patients underwent the following functional tests:

1. Performance-oriented mobility assessment (POMA) [32], which scores 16 items (nine for evaluation of balance, seven for assessment of gait).

2. Outdoor gait assessment [33] recorded with a device (Physilog; BioAGM, Lausanne, Switzerland). Participants were asked to walk, wearing four miniature gyroscopes (ADXRS 250 analogue device) attached to both shanks and thighs with Velcro straps, on a specific walkway at their preferred walking speed. The walkway consisted of two $50 \mathrm{~m}$ tarred pathways, two $50 \mathrm{~m}$ grass pathways and two $20 \mathrm{~m}$ cobblestone pathways in the hospital's backyard. For this study only the tarred terrain was evaluated. A detailed description of this gait assessment has been published elsewhere [33].

3. Dynamic balance test in which participants had to walk as fast and as precisely as possible on a $5 \mathrm{~m}$ beam (15 $\mathrm{cm}$ high, $15 \mathrm{~cm}$ wide). Time was measured with a 
stopwatch. Patients had one practice trial before the test started. If a patient had to step off the beam he or she was allowed to do the test again. In the event that this patient stepped off the beam again, he or she was asked to resume the exercise at the stepping off point, continuing from there to finish the test.

4. Static balance test evaluating postural control by means of a device (Biodex Balance System; Biodex Medical Systems, New York, USA) [34]. The level of difficulty while standing on this platform can be manipulated by altering the resistance of the platform to deviations. The balance test is most difficult when the platform provides the least resistance to tilting and is therefore the least stable. Each participant stands barefoot on the platform and performs two different tests (level $8=$ easiest level, level $6=\mathrm{a}$ more difficult level). The foot position was standardised using a pre-formed triangle (heels together with feet forming an angle of 20 degrees). Patients were instructed to keep their hands at their sides. For safety purposes they were permitted to touch handrails, but only to re-establish balance during extreme postural deviations. Once in this position, the stability platform was unlocked to allow motion. The participants were then instructed to find a position at which they could maintain platform stability. Each recording lasted for $60 \mathrm{~s}$ with a $60 \mathrm{~s}$ rest between each trial. We used a single recording per test condition and only one attempt per condition to reduce fatigue [34]. All tests were done at baseline, after the 12 weeks intervention and at 6 months after the intervention. A balance index was calculated using the time and deviation (in degrees) of the platform away from a level position [35].

Treatment description The training took place twice a week for 60 min over 12 weeks. This intensity was chosen on the basis of previously developed successful interventions in pre-frail elderly persons [25, 36-38]. Each session was conducted in groups (five to eight participants) in order to promote long-term participation [39]. The programme was directed by a physiotherapist and an assistant. Four different physiotherapists and four assistants were trained to direct the sessions in order to guarantee continuity. A session consisted of a warm up ( $5 \mathrm{~min}$ ), followed by a circuit training (40 min) including gait and balance exercises. Each session was composed of a set of ten tasks. Balance and walking tasks (stance on heel/toes, tandem stance, one leg stance, different kinds of walking) alternated with functional strength and endurance exercises (sitting to standing, walking up and down a slope, stair climbing and mini hops). Each task was carried out twice for 1 min each time, and the complexity of the task could be progressively increased, e.g. changing from stable to unstable surfaces (wobble board), increasing step height [40]. Sessions were completed with interactive games (e.g. badminton, obstacle race in teams) (10 $\mathrm{min}$ ) and a short feedback session with suggestions for individual home exercises (5 min). As currently no specific gait and balance programme is offered to diabetic patients, the control group received neither treatment nor specific advice. Both the intervention group and control group were allowed to continue their usual leisure activities.

Sample size calculation In previous studies, gait velocity of diabetic patients was $1.25 \pm 0.19 \mathrm{~m} / \mathrm{s}$ compared with $1.45 \pm$ $0.14 \mathrm{~m} / \mathrm{s}$ in a healthy control group [3]. Allowing that diabetic patients might improve their mean speed from $1.25 \mathrm{~m} / \mathrm{s}$ to $1.35 \mathrm{~m} / \mathrm{s}, 64$ patients were needed to have an $80 \%$ probability that the study would detect a treatment difference at a two sided 5\% level of significance. We assumed a standard deviation of 0.14 of the response variable. A $10 \%$ drop-out rate was hypothesised and therefore we aimed to include 71 patients in total.

Data analysis Statistical analyses were performed using SPSS Version 15 for Windows (SPSS, Chicago, IL, USA). The Student's $t$ test was used to compare baseline similarity. Each outcome was analysed by linear regression (i.e. analysis of covariance). The outcomes at 12 weeks (i.e. post-treatment) and 6 months were dependent variables. The intervention allocation was considered to be a dichotomous independent variable in the analysis. The baseline values of the outcome measures were incorporated in the linear regression model as covariates.

To reduce the risk of Type I error, a Bonferroni corrected alpha level of $p=0.0026$ (alpha divided by number of tests per follow-up) was used to determine a significant difference between groups. However, to allow identification of areas of interest for future investigations and to reduce the risk of Type II errors, the results are presented with the corrected $(p=0.0026)$ and uncorrected $(p=0.05)$ significance levels. An intention to treat analysis was performed and in the event of missing values for any variables, these values were imputed by means of the last observation carried forward method.

\section{Results}

This clinical trial is closed to recruitment and closed to follow-up. The flow chart (Fig. 1) provides a detailed description of drop-outs and loss to follow-up, as well as the number of participants analysed in each group. Exercise adherence ranged from 11 to 24 treatments with a median of 21. Groups were similar at baseline. Descriptive statistics show patients' characteristics (Table 1) and illustrate the 
Fig. 1 Flow chart with detailed description of recruitment, number of drop-outs and reasons for not continuing. The flow chart is based on the CONSORT Statement recommendations in a previous publication [45]

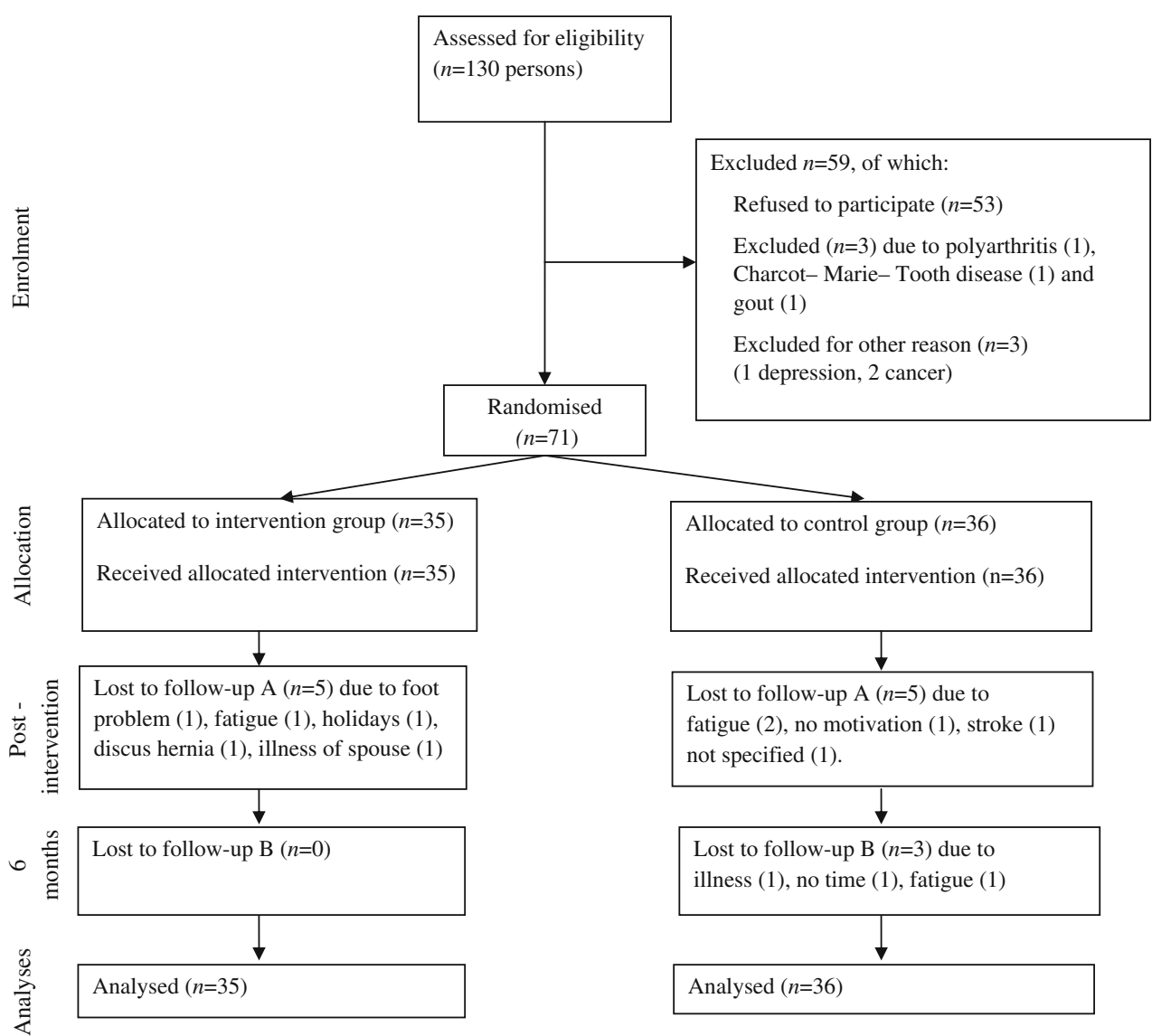

improvement of the intervention group in all variables postintervention (Electronic supplementary material [ESM] Table 1; Fig. 2). Two patients developed pain in their Achilles tendon, obliging us to slow down the progression for 'toe walking' and 'one leg stance' exercises. Intervention group participants partially lost their treatment benefit in the 6 months of follow-up, but their performance level remained superior to that at baseline.

Table 1 Description of patients' baseline characteristics

\begin{tabular}{lcr}
\hline Baseline evaluation & Intervention group & Control group \\
\hline Age (years) & $63(7.99)$ & $64(8.89)$ \\
BMI $\left(\mathrm{kg} / \mathrm{m}^{2}\right)$ & $30.46(6.03)$ & $31.46(5.25)$ \\
Falls in 12 months $(n)^{\mathrm{a}}$ & $0.71(1.07)$ & $0.45(0.89)$ \\
Test with tuning fork & $3.23(1.26)$ & $3.32(1.32)$ \\
Height $(\mathrm{cm})$ & $166.14(8.5)$ & $168.56(8.64)$ \\
Weight $(\mathrm{kg})$ & $83.62(16.56)$ & $89.17(12.33)$ \\
\end{tabular}

Values are mean (SD)

${ }^{\text {a }} \mathrm{A}$ fall was defined as an unexpected event in which the participant comes to rest on the ground, floor or lower level

${ }^{\mathrm{b}}$ Scale $0-8$
Control group patient variables all progressively deteriorated compared with their baseline performance (ESM Table 1).

After intervention, the intervention group increased their habitual walking speed by $0.149 \mathrm{~m} / \mathrm{s}(0.54 \mathrm{~km} / \mathrm{h} ; p<0.001)$ compared with the control group. In addition, a majority of secondary outcome variables showed significant betweengroup differences (at the Bonferroni corrected significance level of $p<0.0026$ ) in favour of the intervention group. These outcome variables were: the dynamic balance test (time to walk over a beam), the POMA test (total score and sub-scores), the Biodex sway index recorded at level 6, the FES-I score, the hip and ankle plantar flexor strength, and hip flexions mobility. After 6 months, the differences in all these variables remained significant $(p<0.0026)$ except for the Biodex sway index $(p=0.005)$ and ankle plantar flexor strength $(p=0.217)($ ESM Table 1$)$.

\section{Discussion}

This study aimed to evaluate the effect of a training programme, which was based on core components for successful fall prevention in the elderly [25], on gait and balance of diabetic patients. Our results confirm that gait 
Fig. 2 Plots illustrating the baseline difference of (a) gait speed, (b) CVGCT, (c) POMA score, (d) time to walk over a beam, (e) Biodex sway index (level 6) and (f) fear of falls for each group at baseline (BA), after the intervention (PI) and at the 6-month follow-up (FU). Values are adjusted mean and $95 \%$ CI. White squares and dotted line, control group; black circles and continuous line, intervention group
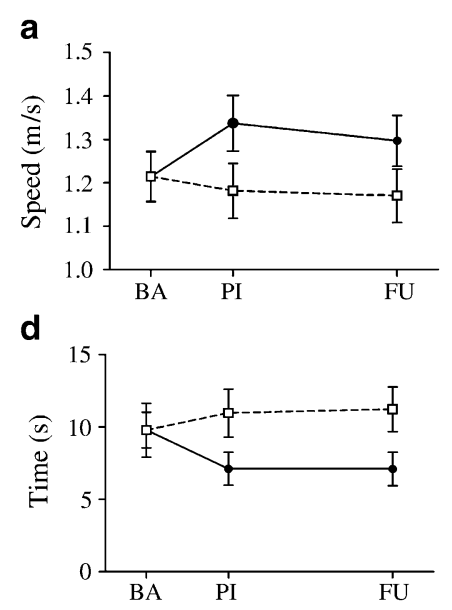


and balance in these patients can be concurrently improved by a targeted intervention. Patients in the intervention group increased gait velocity, balance, muscle strength and joint mobility, and decreased their fear of falling.

However, post intervention evaluation showed no difference between groups for the primary outcome CVGCT (ESM Table 1). Possibly the tarred surface was not challenging enough to fully reveal the gait problems of our diabetic patients. The CVGCT was relatively low at baseline (2.75\% for control group, $2.6 \%$ for intervention group) and consequently did not improve (decrease) after treatment, probably due to a floor effect. Step time variability on smooth and irregular surfaces was recently shown to be most strongly associated with reduction in step length on an irregular surface as compared with a smooth surface [41]. Richardson et al. [41] have shown that the greater the decrease in step length on an irregular surface, the greater the step time variability on both surfaces, and the greater the increase in step time variability on the irregular surface. Thus, analysis on a more challenging surface, e.g. cobblestone pathway, which was beyond this paper's aim, might have revealed differences between groups.

Despite the above, the degree of improvement achieved by the intervention group in gait velocity $(0.54 \mathrm{~km} / \mathrm{h}$ or $11.6 \%$ ) and dynamic balance (time to walk over a beam) (3.39 s or $34 \%$ ) was not only significant but also clinically relevant. A decrease in gait speed of $0.1 \mathrm{~m} / \mathrm{s}$ in the elderly has been associated with a $10 \%$ decrease in the ability to perform daily living activities [42]. Balance tests underscored our patients' progress. At baseline our population scored 23 to 24 of 28 possible points on the POMA. These values are just within the critical range (19 to 24 points) that implies a moderate risk of falling [43]. Intervention group patients passed this moderate risk of falling cut-off point after the training. In addition, their change of 2.0 points signifies a real improvement considering that effects greater than 0.8 (with a group size of $n=30$ ) reputedly represent a significant improvement unrelated to chance fluctuations [43]. The more difficult Biodex balance test (level 6) also highlighted patients' progress in postural control. The sway index decrease of 1.9 at level 6 represents a $31 \%$ improvement.

Importantly, all gait and balance variables (except the Biodex balance test) remained significant at 6-month follow-up, although some treatment benefit was lost. Increased hip and ankle strength as well as ankle mobility may explain the progress in gait velocity and both static and dynamic balance, although the improvement of ankle dorsal flexor strength and ankle dorsiflexion mobility just failed to be significant at the Bonferroni corrected significance level. These two values further decreased at 6 months, possibly explaining the regression of gait and balance measures. Knee flexion mobility showed normal baseline values, thereby explaining the absence of improvement after training. We decided not to calculate the post-treatment group effect on knee and hip extension mobility as none of the patients had a flexor contracture.

Regarding the FES-I, patients showed a relatively low level of concern about falling. Perhaps this result reflects our study population's relatively good gait function and functional capacity. Apparently, our participants had moderate diabetes, with on average only minimal neuropathy. This could have been influenced by the inclusion and exclusion criteria used and must be considered in the clinical decision-making process. It may be hypothesised that patients with more severe diabetic peripheral neuropathy or with more impaired functional capacity would benefit less from training, due to the fact that, from a functional point of view, the detrimental effects of the disease are impossible to reverse or compensate. However, the reverse may also be possible. Patients suffering from more severe peripheral neuropathy could benefit even more from a structured exercise regimen, as their condition provides more scope for improvement. Moreover, even though balance and gait changes seen in diabetic patients are probably primarily due to neuropathy, there are other 
potential contributors [5]. Gait impairments are also observed in diabetic patients without clinically detectable neuropathy [3], raising the question of whether it would be worthwhile to test a similar, but more intensive programme on diabetic patients without neuropathy in order to prevent further aggravation of gait and balance.

All exercises were taught by qualified physiotherapists and were function-orientated. To boost patient motivation and enable them to interact with other patients, the programme was carried out in small groups at the hospital's gymnasium. For further discussion of the results, the effect of our primary outcome was compared with the results of a meta-analysis evaluating the effect of exercise on gait speed in the elderly [22]. This meta-analysis reported a success rate of $57 \%$ for exercise training on habitual gait speed. The overall gait speed change reported was $0.01 \mathrm{~m} / \mathrm{s}$ compared with our $0.15 \mathrm{~m} / \mathrm{s}$. However, the range of velocity change was large $(-0.2$ to $0.34 \mathrm{~m} / \mathrm{s})$, which could be due to the various studies included and the evaluation of populations with diverse co-morbidities under varied conditions (e.g. $6 \mathrm{~m}$ distance compared with a 6 min walk). We further compared our baseline CVGCT with values reported for elderly patients. The values of our population (control group $2.6 \%$, intervention group $2.75 \%$ ) are between those reported for elderly non-fallers $(2.1 \%$; mean age 76 years) and those for elderly fallers (3.8\%; mean age 82 years) [44], which was somewhat better than expected. However, this may be explained by the fact that our population was about 15 years younger than participants in Hausdorff's trial [44].

Despite the beneficial effect of our programme on gait speed and balance, its influence on fall frequency in diabetic patients still needs to be properly assessed. Although the CVGCT did not improve, our results provide encouraging data, which justify further studies with larger sample sizes focusing on fall frequency itself, physical activity level and quality of life.

Our baseline evaluation contains no information about patients' cardiac status, severity of retinopathy, visual acuity, disease duration, orthostatic hypotension, medications or other possible factors related to gait and balance impairments, and to treatment responsiveness. Nevertheless, we assume that randomisation balanced these potential confounders between groups.

The fact that the control group was given no attention at all may represent a confounder for this study's results. The degree to which an improvement depends on personal attention from the therapists, vs the effect of the actual exercise regimen, needs to be considered. However, the positive result on all measured outcomes clearly points to a beneficial effect due to therapeutic exercise.

Muscle strength was measured with a hand-held dynamometer, which gives a more precise measurement than manual muscle testing. However, the reliability and accuracy of the measurement may be limited by the investigator's ability to hold the dynamometer stationary and by the fact that participants may overpower the testers. We tried to minimise this problem by ensuring that the same person always carried out the tests. Nevertheless, the relatively low ratio between ankle plantar flexor and ankle dorsal flexor strength could indicate an underestimation of plantar flexor strength. Further investigations should address this limitation. In addition, not only sagittal but also frontal plane muscle strength (i.e. hip abductors and adductors), which is involved in frontal plane stability during gait, should be evaluated.

Although our study showed positive results, clinicians should be aware of possible adverse events. Two patients developed pain in their Achilles tendon, obliging us to slow down the progression for 'toe walking' and 'one leg stance' exercises. More moderate progression and a longer warm up could possibly avoid such incidents.

To the best of our knowledge, this is one of the first randomised controlled trials to describe an effective physiotherapy training programme geared to concurrently improving the balance and gait of diabetic patients. Future studies should examine the effect of exercise regimens on patient groups differentiated by neuropathy status (patients without, with mild or with severe peripheral neuropathy, identified by a more complex instrument for peripheral neuropathy screening). In addition, outcomes such as functional capacity, the number of falls or physical activity level should be considered in order to draw meaningful conclusions about exercise efficacy among patients with diabetes, thereby facilitating medical and clinical decision-making. The appraisal of (1) advantages, difficulties and feasibility of treatment, and/or (2) prevention of gait and balance problems, as well as (3) the related fall risk in diabetic patients may be another interesting issue for further quantitative and qualitative studies.

\section{Conclusion}

A specific gait and balance training programme based on a circuit approach and including gait and balance exercises combined with function-orientated strengthening can improve gait speed and balance, and increase both muscle strength and joint mobility of diabetic patients with a vibration perception threshold $\leq 4$. Further studies with a larger sample size are needed to explore the influence of these improvements on the number of reported falls, patients' physical activity levels and quality of life.

Acknowledgements We would like to thank V. Barthassat and S. Thalmann (University Hospital of Geneva, Switzerland) for their help during the recruitment, B. le Callennec (EPFL, Lausanne, Switzerland) for data analysis, P. Sclison (University Hospital of 
Geneva, Switzerland) for linguistic revision and all physiotherapists of the Department of Neurosciences at the University Hospital of Geneva, Switzerland for their cooperation. We also thank all patients for their participation.

Duality of interest The authors declare that there is no duality of interest associated with this manuscript.

Open Access This article is distributed under the terms of the Creative Commons Attribution Noncommercial License which permits any noncommercial use, distribution, and reproduction in any medium, provided the original author(s) and source are credited.

\section{References}

1. Dyck PJ, Kratz KM, Karnes JL et al (1993) The prevalence by staged severity of various types of diabetic neuropathy, retinopathy, and nephropathy in a population-based cohort: the Rochester Diabetic Neuropathy Study. Neurology 43:817-824

2. Ziegler D, Gries FA, Spuler M, Lessmann F (1992) The epidemiology of diabetic neuropathy. Diabetic Cardiovascular Autonomic Neuropathy Multicenter Study Group. J Diabetes Its Complicat 6:49-57

3. Allet L, Armand S, de Bie RA et al (2009) Gait alterations of diabetic patients while walking on different surfaces. Gait Posture 29:488-493

4. Allet L, Armand S, Golay A, Monnin D, de Bie R, de Bruin ED (2008) Gait characteristics of diabetic patients: a systematic review. Diabetes Metab Res Rev 24:173-191

5. Goldberg A, Russell JW, Alexander NB (2008) Standing balance and trunk position sense in impaired glucose tolerance (IGT)related peripheral neuropathy. J Neurol Sci 270:165-171

6. Bonnet C, Carello C, Turvey MT (2009) Diabetes and postural stability: review and hypotheses. J Mot Behav 41:172-190

7. Kwon OY, Minor SD, Maluf KS, Mueller MJ (2003) Comparison of muscle activity during walking in subjects with and without diabetic neuropathy. Gait Posture 18:105-113

8. Mueller MJ, Minor SD, Sahrmann SA, Schaaf JA, Strube MJ (1994) Differences in the gait characteristics of patients with diabetes and peripheral neuropathy compared with age-matched controls. Phys Ther 74:299-308

9. Lafond D, Corriveau H, Prince F (2004) Postural control mechanisms during quiet standing in patients with diabetic sensory neuropathy. Diabetes Care 27:173-178

10. Nardone A, Grasso M, Schieppati M (2006) Balance control in peripheral neuropathy: are patients equally unstable under static and dynamic conditions? Gait Posture 23:364-373

11. Turcot K, Allet L, Golay A, Hoffmeyer P, Armand S (2009) Investigation of standing balance in diabetic patients with and without peripheral neuropathy using accelerometers. Clin Biomech 24:716-721

12. Simoneau GG, Ulbrecht JS, Derr JA, Becker MB, Cavanagh PR (1994) Postural instability in patients with diabetic sensory neuropathy. Diabetes Care 17:1411-1421

13. Maurer MS, Burcham J, Cheng H (2005) Diabetes mellitus is associated with an increased risk of falls in elderly residents of a longterm care facility. J Gerontol A Biol Sci Med Sci 60:1157-1162

14. Richardson JK, Sandman D, Vela S (2001) A focused exercise regimen improves clinical measures of balance in patients with peripheral neuropathy. Arch Phys Med Rehabil 82:205-209

15. Pataky Z, Vischer U (2007) Diabetic foot disease in the elderly. Diabetes Metab 33(Suppl 1):S56-S65
16. Petrofsky J, Lee S, Cuneo ML (2005) Gait characteristics in patients with type 2 diabetes; improvement after administration of rosiglitazone. Med Sci Monit 11:PI43-PI51

17. Richardson JK, Thies SB, DeMott TK, Ashton-Miller JA (2004) Interventions improve gait regularity in patients with peripheral neuropathy while walking on an irregular surface under low light. J Am Geriatr Soc 52:510-515

18. Nissen SE, Wolski K (2007) Effect of rosiglitazone on the risk of myocardial infarction and death from cardiovascular causes. $\mathrm{N}$ Engl J Med 356:2457-2471

19. Tsang T, Orr R, Lam P, Comino EJ, Singh MF (2007) Health benefits of Tai Chi for older patients with type 2 diabetes: the "Move It For Diabetes study"-a randomized controlled trial. Clin Interv Aging 2:429-439

20. Orr R, Tsang T, Lam P, Comino E, Singh MF (2006) Mobility impairment in type 2 diabetes: association with muscle power and effect of Tai Chi intervention. Diabetes Care 29:2120-2122

21. Steadman J, Donaldson N, Kalra L (2003) A randomized controlled trial of an enhanced balance training program to improve mobility and reduce falls in elderly patients. J Am Geriatr Soc 51:847-852

22. Lopopolo RB, Greco M, Sullivan D, Craik RL, Mangione KK (2006) Effect of therapeutic exercise on gait speed in communitydwelling elderly people: a meta-analysis. Phys Ther 86:520-540

23. de Bruin ED, Murer K (2007) Effect of additional functional exercises on balance in elderly people. Clin Rehabil 21:112-121

24. Hill-Westmoreland EE, Soeken K, Spellbring AM (2002) A metaanalysis of fall prevention programs for the elderly: how effective are they? Nurs Res 51:1-8

25. Gillespie LD, Gillespie WJ, Robertson MC, Lamb SE, Cumming RG, Rowe BH (2003) Interventions for preventing falls in elderly people. Cochrane Database Syst Rev:CD000340

26. Hausdorff JM, Rios DA, Edelberg HK (2001) Gait variability and fall risk in community-living older adults: a 1-year prospective study. Arch Phys Med Rehabil 82:1050-1056

27. Barnett A, Smith B, Lord SR, Williams M, Baumand A (2003) Community-based group exercise improves balance and reduces falls in at-risk older people: a randomised controlled trial. Age Ageing 32:407-414

28. Allet L, Armand S, de Bie RA et al (2009) Clinical factors associated with gait alterations in diabetic patients. Diabet Med 26:1003-1009

29. Kastenbauer T, Sauseng S, Brath H, Abrahamian H, Irsigler K (2004) The value of the Rydel-Seiffer tuning fork as a predictor of diabetic polyneuropathy compared with a neurothesiometer. Diabet Med 21:563-567

30. Morey JK, Joshua AC (2005) Strength testing using hand-held dynamometry. Phys Ther Rev 10:99-112

31. Yardley L, Beyer N, Hauer K, Kempen G, Piot-Ziegler C, Todd C (2005) Development and initial validation of the Falls Efficacy Scale-International (FES-I). Age Ageing 34:614-619

32. Tinetti ME (1986) Performance-oriented assessment of mobility problems in elderly patients. J Am Geriatr Soc 34:119-126

33. Allet L, Armand S, de Bie RA et al (2008) Reliability of diabetic patients' gait parameters in a challenging environment. Gait Posture 28:680-686

34. Arnold BL, Schmitz RJ (1998) Examination of balance measures produced by the Biodex Stability System. J Athl Train 33:323327

35. Swanik CB, Lephart SM, Rubash HE (2004) Proprioception, kinesthesia, and balance after total knee arthroplasty with cruciateretaining and posterior stabilized prostheses. J Bone Joint Surg Am 86-A:328-334

36. Barrett CJ, Smerdely P (2002) A comparison of community-based resistance exercise and flexibility exercise for seniors. Aust J Physiother 48:215-219 
37. Faber MJ, Bosscher RJ, Chin APMJ, van Wieringen PC (2006) Effects of exercise programs on falls and mobility in frail and prefrail older adults: a multicenter randomized controlled trial. Arch Phys Med Rehabil 87:885-896

38. Rubenstein LZ, Josephson KR, Trueblood PR et al (2000) Effects of a group exercise program on strength, mobility, and falls among fallprone elderly men. J Gerontol A Biol Sci Med Sci 55:M317-M321

39. Estabrooks PA (2000) Sustaining exercise participation through group cohesion. Exerc Sport Sci Rev 28:63-67

40. Robertson MC, Campbell AJ, Gardner MM, Devlin N (2002) Preventing injuries in older people by preventing falls: a metaanalysis of individual-level data. J Am Geriatr Soc 50:905-911

41. Richardson JK, Thies S, Ashton-Miller JA (2008) An exploration of step time variability on smooth and irregular surfaces in older persons with neuropathy. Clin Biomech (Bristol, Avon) 23:349-356
42. Judge JO, Schechtman K, Cress E (1996) The relationship between physical performance measures and independence in instrumental activities of daily living. The FICSIT Group. Frailty and Injury: Cooperative Studies of Intervention Trials. J Am Geriatr Soc 44:1332-1341

43. Faber MJ, Bosscher RJ, van Wieringen PC (2006) Clinimetric properties of the performance-oriented mobility assessment. Phys Ther 86:944-954

44. Hausdorff JM, Edelberg HK, Mitchell SL, Goldberger AL, Wei JY (1997) Increased gait unsteadiness in community-dwelling elderly fallers. Arch Phys Med Rehabil 78:278-283

45. Boutron I, Moher D, Altman DG, Schulz K, Ravaud P, for the CONSORT group (2008) Methods and processes of the CONSORT Group: example of an extension for trials assessing non pharmacologic treatments. Ann Intern Med 148:W60-W67 